\title{
Xanthogranulomatous funiculitis and epididymo-orchitis in a tetraplegic patient
}

\author{
S Vaidyanathan ${ }^{*, 1}$, P Mansour ${ }^{2}$, KF Parsons ${ }^{4}$, G Singh ${ }^{1}$, BM Soni ${ }^{1}$, R Subramaniam ${ }^{3}$, T Oo ${ }^{1}$ and P Sett ${ }^{1}$ \\ ${ }^{1}$ Regional Spinal Injuries Centre, Southport and Formby District General Hospital, Southport and Ormskirk Hospital \\ NHS Trust, Southport, Merseyside PR8 6PN, UK; ${ }^{2}$ Department of Cellular Pathology, Southport and Formby \\ District General Hospital, Southport and Ormskirk Hospital NHS Trust, Southport, Merseyside PR8 6PN, UK; \\ ${ }^{3}$ Department of Radiology, Southport and Formby District General Hospital, Southport and Ormskirk Hospital NHS \\ Trust, Southport, Merseyside PR8 6PN, UK; ${ }^{4}$ Department of Urology, Royal Liverpool University Hospital, Prescot \\ Street, Liverpool L7 8XP, UK
}

\begin{abstract}
Study design: A case report of xanthogranulomatous funiculitis and epididymo-orchitis. Xanthogranulomatous inflammation is an uncommon, non-neoplastic process characterised by destruction of tissue, which is replaced by a striking cellular infiltrate of lipid-laden macrophages.

Case report: A 21-year male sustained complete tetraplegia at C-6 level, after a fall in 1998 . The neuropathic bladder was managed with an indwelling urethral catheter. He had many unsuccessful trials of micturition. Sixteen months after the cervical injury, he noticed swelling of the left side of the scrotum following removal of a blocked catheter. He was prescribed antibacterial therapy. Four weeks later, physical examination revealed a hard and irregular swelling encompassing the testis, epididymis and spermatic cord. The clinical diagnosis was epididymo-orchitis progressing to pyocele. Through a scrotal incision, the swollen testis, epididymis and diseased segment of the spermatic cord were removed en masse. Histopathology showed extensive areas of necrosis, with xanthogranulomatous inflammation in the spermatic cord and to a lesser extent in the testis/epididymis.

Conclusion: Repeated episodes of high-pressure urinary reflux along the vas deferens during dyssynergic voiding, and subsequent interstitial extravasation of urine together with chronic, low-grade, suppurative infection possibly led to development of xanthogranulomatous inflammation in the testis and the epididymis. Since tissue destruction is a feature of xanthogranulomatous inflammation, the definite and curative treatment is either complete (or, where applicable, partial) excision of the affected organ in most of the cases.
\end{abstract}

Spinal Cord (2000) 38, $769-772$

Keywords: xanthogranulomatous inflammation; spinal cord injury; funiculitis; epididymoorchitis; neuropathic bladder

\section{Introduction}

Xanthogranulomatous inflammation is an uncommon, non-neoplastic process characterised by destruction of tissue, which is replaced by a striking cellular infiltrate of lipid-laden macrophages. ${ }^{1}$ Microscopically, the lesion consists of a dominant infiltrate of foamy macrophages interspersed with a minor component of lymphocytes and plasma cells. ${ }^{2}$ The aetiology of xanthogranulomatous lesions may include immunological defects, chronic infection, abnormal lipid accumulation, and reduction in chemotactic activities. $^{3}$ Xanthogranulomatous inflammation has been

*Correspondence: S Vaidyanathan, Regional Spinal Injuries Centre, District General Hospital, Town Lane, Southport, Merseyside PR8 6PN, UK described commonly in the kidney, but can occur in other parts of the body such as gall bladder, ovary, appendix, and urinary bladder. ${ }^{3}$ Solitary cases of xanthogranulomatous orchitis ${ }^{1}$ and epididymitis ${ }^{2}$ have been reported. Xanthogranulomatous funiculitis has not thus far been reported.

In spinal cord injury patients, xanthogranulomatous inflammation of the kidney, ${ }^{4}$ and the perineum ${ }^{5}$ have been published. We report a tetraplegic patient in whom the xanthogranulomatous inflammation was seen predominantly in the spermatic cord, and to a lesser extent in the ipsilateral testis/epididymis. To our knowledge, simultaneous occurrence of xanthogranulomatous funiculitis and xanthogranulomatous epididymo-orchitis has not been described. 


\section{Case report}

A 21-year male sustained fracture of C-5 with complete tetraplegia at the C-6 level, after a fall in 1998. The neuropathic bladder was managed by an indwelling size 14 Fr. Foley catheter. Recurrent urinary tract infections ensued. Sixteen months after the cervical injury, he developed left hemiscrotal swelling. With a diagnosis of acute epididymo-orchitis, he was prescribed ciprofloxacin intravenously. Subsequently, he received a course of gentamicin $240 \mathrm{mg}$ intravenously once a day and ciprofloxacin $500 \mathrm{mg}$ twice a day by mouth. Urine microbiology showed no growth of organism. Three to four weeks later, the swelling ulcerated in its central part and he was admitted to the spinal unit for further management. Physical examination revealed a hard and irregular swelling encompassing the testis, epididymis and spermatic cord on the left side. The swelling had ulcerated in its most prominent part. The surrounding area was soft and fluctuant. Scrotal ultrasound revealed $3 \times 1 \mathrm{~cm}$ collection in the left side of the scrotum. (Figure 1A and B). The clinical diagnosis was epididymo-orchitis progressing to pyocele. Through a scrotal incision, the swollen testis, epididymis and diseased segment of the spermatic cord were removed en masse. Microbiology of the pus showed no significant growth.

On gross examination, the testis and epididymis were no longer separately identifiable. Histopathological examination confirmed severe epididymo-orchitis, with extensive areas of necrosis. In some areas, particularly within the spermatic cord, the inflammation had a xanthogranulomatous appearance, with sheets of foamy macrophages replacing areas of granulation tissue. These xanthogranulomatous areas were vascular and contained an inconspicuous population of lymphocytes, plasma cells and occasional eosinophils. (Figure 2A). Michaelis-Gutmann bodies were absent. Marked fibrosis in the surrounding tissue confirmed the chronic nature of the inflammatory process. Xanthogranulomatous inflammation was also present to a lesser extent in the testis/ epididymis (Figure 2B). There was no evidence of neoplasia.

\section{Discussion}

\section{Pathogenesis of xanthogranulomatous inflammation}

Histologically, the xanthogranulomatous lesion consisted of foam cells and lymphocytes. With respect to the origin of foam cells, Nakashiro and associates ${ }^{6}$ were of the opinion that the vast majority of foam cells were derived from monocytes/macrophages because they were positive for KP1, HAM56, CD11b, and CD68. They found many T lymphocytes interspersed among macrophage foam cells. The subtyping of $\mathrm{T}$ cells indicated a heterogenous population composed of both CD4+ and CD8+ lymphocytes typically in a ratio of $1: 2$. These authors concluded

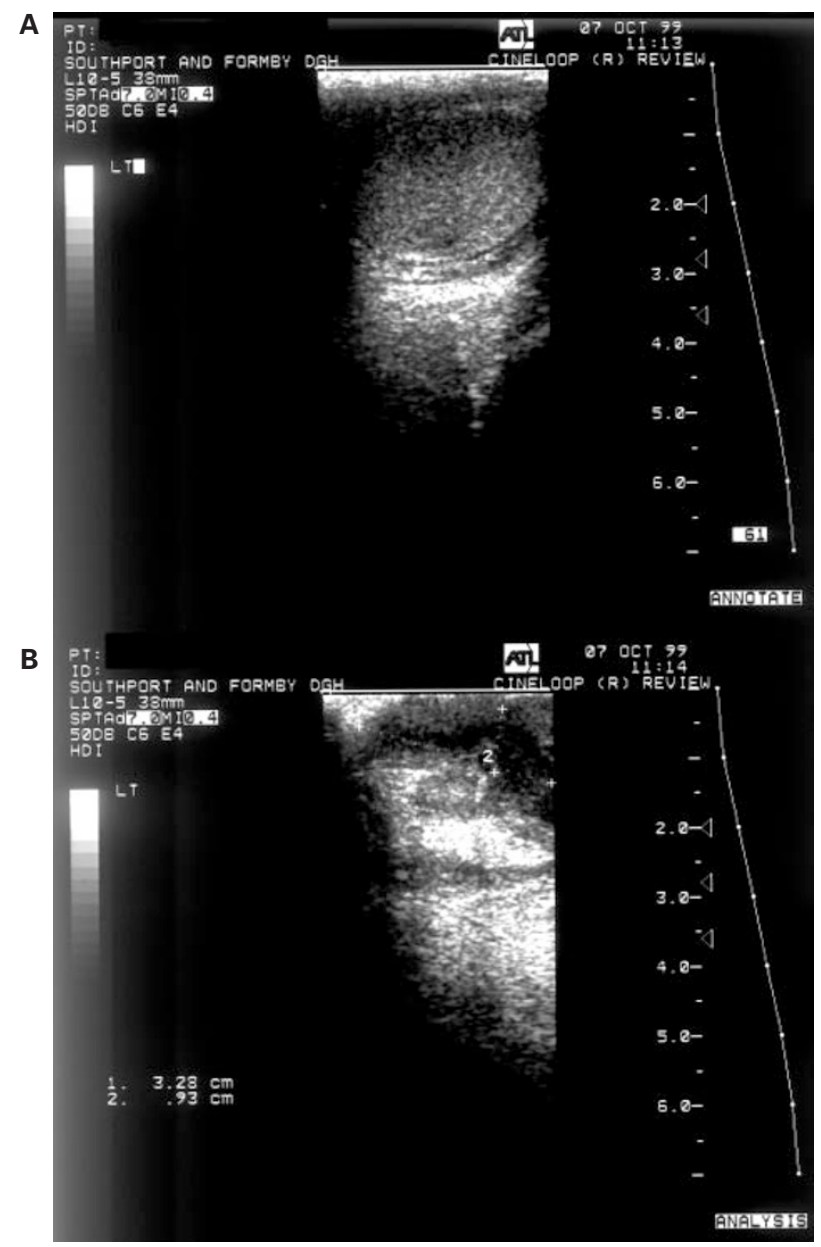

Figure 1 Ultrasound scan of the left testis and epididymis: (A) The testicular parenchyma is echo-poor, compatible with orchitis. (B) The epididymis is enlarged. There is fluid collection measuring $3 \times 1 \mathrm{~cm}$ containing debris, which is suggestive of abscess

that xanthogranulomatous inflammation is a disorder characterised by accumulation of macrophage foam cells and $\mathrm{T}$ cells. Delayed type hypersensitivity reaction of cell-mediated immunity may also be implicated in the pathogenesis of xanthogranulomatous inflammation. ${ }^{6}$

Many infective microorganisms have been reported to have roles in the aetiology of the xanthogranulomatous process. The relationship of Bacteroides and Peptostreptococcus species with xanthogranulomatous pyelonephritis has been described. In the case reported by Tai and associates, ${ }^{3} B$. fragilis and $E$. coli were cultured from the area of necrosis and abscess in the centre of the mass, which strongly supported the relationship of anaerobes with xanthogranulomatous cystitis. In xanthogranulomatous cholecystitis, bacterial antigens were demonstrated by immunohistochemistry using a polyclonal anti-E. coli antibody. The time period after the initial clinical manifestation was critical for identifying the bacterial antigens in the 
A

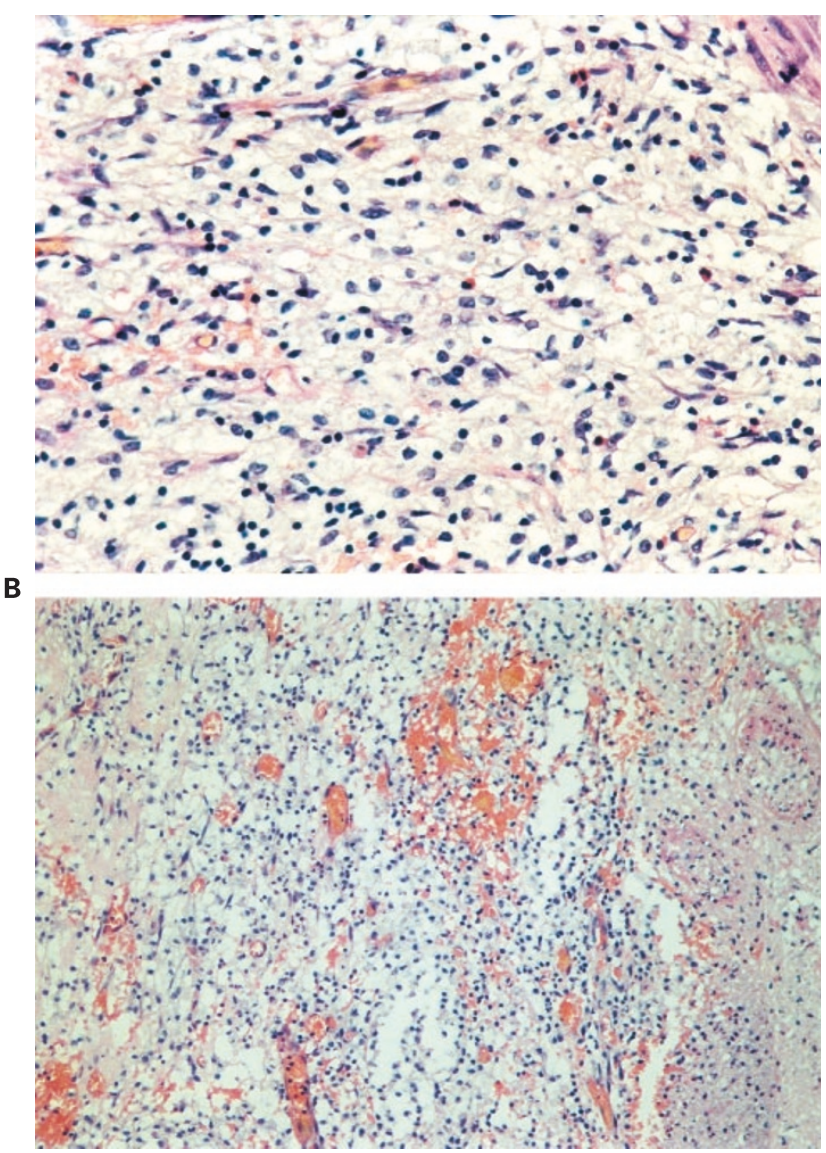

more granular, eosinophilic and less vacuolated cytoplasm (von Hansemann cells). The MichaelisGutmann bodies represent the characteristic round intracellular and extracellular inclusions in the macrophages. These inclusions are composed of bacterial debris and they are thought to reflect defective macrophage function.

In the kidney, xanthogranulomatous pyelonephritis can be virtually indistinguishable (both grossly and microscopically) from renal cell carcinoma; immunohistochemistry may be required to clarify the diagnosis.

Pathogenesis of xanthogranulomatous epididymo-orchitis in spinal cord injury patients

The risk factors for development of epididymo-orchitis are:

(1) Reflux of urine to the seminal vesicles or vas. ${ }^{8}$ In tetraplegic patients with detrusor-external sphincter dyssynergia, high-pressure reflux of urine into the vas and scrotum is more likely to occur during dyssynergic voiding.

(2) Indwelling urethral catheter drainage. Natural-fill urodynamics in chronically catheterised patients with spinal cord injury revealed that an indwelling catheter on free drainage was no guarantee of a constantly low intravesical pressure, and phasic bladder contractions occurred despite catheter drainage. ${ }^{9}$ Detrusor contractions stimulated by the catheter, and consequent striated sphincter dyssynergia could predispose to urethro-vasal reflux of infected urine in a patient with an indwelling urinary catheter.

Both these risk factors were present in this tetraplegic patient. He was on long-term indwelling urethral catheter drainage, but had had catheter-free trials of voiding. He developed sweating and headaches during reflex voiding. The ultrasound scan showed $>200 \mathrm{ml}$ of urine in the bladder. Therefore, indwelling urethral catheter drainage was re-established. The present episode of scrotal swelling began soon after removal of a blocked catheter. Subsequently, reflex voiding was established for a short period. He then noticed swelling of the left side of the scrotum. This observation suggested that urethro-vasal reflux of urine was likely during the dyssynergic voiding episodes. It is possible that repeated episodes of high-pressure urinary reflux along the vas deferens and subsequent interstitial extravasation of urine together with chronic, lowgrade, suppurative infection possibly leads to development of xanthogranulomatous inflammation in the testis and the epididymis. In an analogous situation, extravasation of bile in the interstitial tissue has been postulated to be a predisposing factor for xanthogranulomatous inflammation of the gall bladder. ${ }^{6}$ 
Prevention of epididymo-orchitis in spinal cord injury patients

The strategy for prevention of epididymo-orchitis in spinal cord injury patients should include the following principles:

(1) Prompt treatment of urinary tract infection with adequate doses of appropriate antibacterial agent based on microbiological cultures and susceptibility tests.

(2) Avoidance where possible, of long-term indwelling urethral catheter drainage.

(3) The preferred method of managing neurogenic bladder is by:

(i) Intermittent catheterisation using sterile, selflubricating catheters that cause less friction in the urethra.

(ii) Adjuvant pharmacotherapy to decrease high intravesical pressures - e.g. oxybutynin, or tolterodine.

(4) Lessening of the resistance distal to the ejaculatory duct by sphincterotomy of the distal urethral sphincter muscle in selected patients.

\section{Treatment of xanthogranulomatous inflammation}

Since tissue destruction is a feature of xanthogranulomatous inflammation, the definite and curative treatment is either complete or, where applicable, partial surgical excision of the affected organ in most of the cases. Antimicrobial therapy for anaerobic infection is recommended, as anaerobes may be important in the pathogenesis of xanthogranulomatous inflammation.

Rarely, antibiotic therapy may result in complete cure of the xanthogranulomatous inflammation. Mollier and associates ${ }^{10}$ described a case of xanthogranulomatous pyelonephritis in a young woman in whom total renal recovery was achieved by antibiotic treatment alone. Brown and associates ${ }^{11}$ reported a case of focal xanthogranulomatous pyelonephritis in a child, successfully cured with antibiotics only. The standard treatment for xanthogranulomatous inflammation has been either total or, when applicable, partial surgical excision of the affected organ. However, recent reports of the successful medical management of focal xanthogranulomatous pyelonephritis suggest that a trial of antibiotics prior to surgery is warranted in cases of xanthogranulomatous inflammation affecting only a segment of an organ.

In our patient, ultrasound scan of the scrotum showed an abscess and histology confirmed the presence of extensive areas of necrosis in the testis and epididymis. Therefore, medical therapy with antibiotics alone was not a viable option. In this tetraplegic patient, excision of the testis, epididymis, and diseased segment of the spermatic cord en masse, combined with a course of metronidazole led to a satisfactory outcome.

\section{References}

1 Usamentiaga E et al. Xanthogranulomatous orchitis. Urology 1998; 52: $891-892$.

2 Wierner LB, Riehl PA, Baum N. Xanthogranulomatous epididymitis: a case report. J Urol 1987; 138: $621-622$.

3 Tai H-L, Chen C-C, Yeh K-T. Xanthogranulomatous cystitis associated with anaerobic bacterial infection. J Urol 1999; 162: $795-796$.

4 Goswami AK, Suryaprakash B, Malik AK, Vaidyanathan S. Development of fatal bilateral xanthogranulomatous pyelonephritis in a paraplegic patient: case report. Paraplegia 1988; 26: $62-65$.

5 Belville WD, Park JM, Kelly JL, Vaccaro JA. Perineal xanthogranulomatous pseudotumor due to intermittent catheterisation: a liposarcoma mimic. Case report. Paraplegia 1994; 32: $624-626$

6 Nakashiro $\mathrm{H}$ et al. Xanthogranulomatous cholecystitis. Cell composition and a possible pathogenetic role of cell-mediated immunity. Path Res Pract 1995; 191: $1078-1086$.

7 Mori M, Watanabe M, Sakuma M, Tsutsumi Y. Infectious etiology of xanthogranulomatous cholecystitis: immunohistochemical identification of bacterial antigens in the xanthogranulomatous lesions. Pathology International 1999; 49: 849-852.

8 Ahn JH, Reiter R, Farcon E, Morales P. Scrotal fistula from urethro-vasal reflux in paraplegia. Urology 1989; 34: 383-384.

9 Jamil F, Williamson M, Ahmed YS, Harrison SCW. Natural-fill urodynamics in chronically catheterized patients with spinal cord injury. Br J Urol 1999; 83: 396-399.

10 Mollier S et al. Pseudoneoplastic xanthogranulomatous pyelonephritis. A typical clinical presentation but unusual diagnosis and treatment. Eur Urol 1995; 27: 170-3.

11 Brown Jr PS, Dodson M, Weintrub PS. Xanthogranulomatous pyelonephritis: report of non-surgical management of a case and review of the literature. Clin Infect Dis 1996; 22: 308-314. 